\title{
Design of Decision Support System for Mechanical Conservation Tillage
}

\author{
Junjing Yuan ${ }^{1}$, Jian Zhang ${ }^{2, *}$, and Hongzhen $\mathrm{Cai}^{3}$ \\ ${ }^{1}$ Beijing Vocational College of Electronic Science, Beijing, 100176, China \\ Yuan.junjing@163.com \\ ${ }^{2}$ Beijing Information Science \& Technology University, Beijing, 100085, China \\ Zhj001@cau.edu.ch \\ ${ }^{3}$ Shandong University of Technology, Zibo, 255091, China \\ chzhesdut.edu.cn
}

\begin{abstract}
This paper designed decision support system for mechanical conservation tillage, which includes three subsystems, such as subsystem of development potential estimation, subsystem of optimum model or suitable models selection, and subsystem of economy benefit estimation. In different area, it is necessary to alter, increase or decrease some evaluation index to accommodate the agricultural production. This decision support system is based on the ordinary technique such as .NET Framework and SQL Server 2005, which includes three kinds of function and four levels.
\end{abstract}

Keywords: decision support system, subsystem, evaluation index.

\section{Introduction}

Mechanical conservation tillage is a new agricultural production mode [1-2], and the development of this tillage is becoming the national economy and the people's livelihood issues stage by stage. How to spread mechanical conservation tillage effectively in China is a difficulty. As an assistant decision-making means [3-4], the decision support system for experimentation model of mechanical conservation tillage is focused by the relative organizations. According to the characteristics of investigation area, production practices, projection pursuit, fuzzy comprehensive evaluation and dynamic estimation of economic benefits $[1,2,5]$, this paper designed the experimentation model system for mechanical conservation tillage and develops the decision support system. With the help of the decision support system, it is much easier to finish the estimation of spreading, the selection of experimentation model and the dynamic estimation of economic benefits with low cost and high efficiency.

\section{Demand Analysis}

In the program of popularization and actualization of new tillage, the decision support system is an available tool to solve some problems. The details are as follows.

\footnotetext{
* Corresponding author.
} 
(1) The design of system is an available approach to the popularization or farm examination of mechanical conversation tillage in the double-crop one year regions;

(2) In the system, it is necessary to construct the databank, model bank and knowledge bank, which are the foundation of memory, query, renovation and management;

(3) The system is useful to conform the degree of difficulty in the program of examination or popularization of the mechanical conservation tillage;

(4) The system is helpful to conform the suitable model of the optimum model by comparing the situation of field, technology, and tillage approach;

(5) The system is available to analyze the sensitivity and estimate the economic benefits dynamically;

(6) The functions the decision support system, such as renovation of databank, extension of system and improvement of model, should be convenient and rapid.

The decision support system for mechanical conservation tillage in the double-crop one year regions is an effective and assistant tool to solve some problems, and the system includes the following three levels, the first level provides some relative data and information to the decision-maker and analyzes the data and information, the second level is focus on the decision problems and draw out the decision program, the third level is to analyze and estimate the decision program by the human-computer interaction.

\section{System design [6]}

In the program of designing the decision support system, the designer should abide by the following three principles. Firstly, practicability is the basic principal of the system, which makes the system functions and decision demand matching. Secondly, the system is easy to learn and use, and the friendly human-computer interaction is required. Thirdly, the favorable expansions and maintenances of system should be taken into account.

\subsection{System Structure}

Agricultural production involved many factors and the randomicity of some indexes determined the complexity of the system. This paper designs a decision support system, which is a visual software and includes some functions, such as data analysis, potential evaluation, adaptability estimation and model selection, economic benefit estimation and sensitivity analysis, and so on. Fig. 1 describes the main structure of the system.

\subsection{System Flow}

The decision support provides the basic function, such as file management, information query, statistics analysis, user management, system help, result output and printing, etc. In this paper, the author designs the system according to the project theory, and the system development flow is described in Fig. 2. 


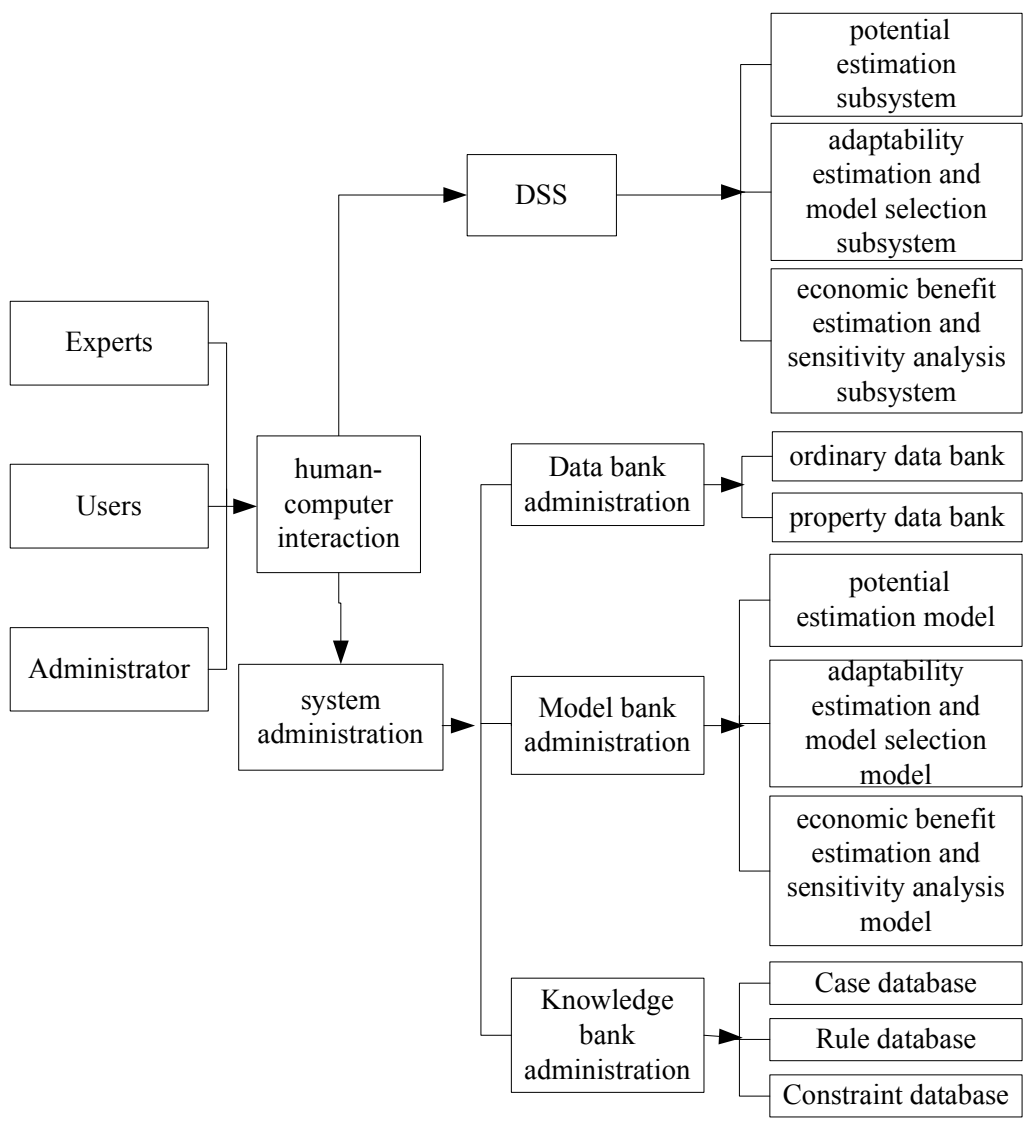

Fig. 1. Structure of decision support system

\subsection{System Frame}

The decision support system is on the bases of data collection and science management, and the large volume of data and the complex interface are difficult problems in the program of constructing the system successfully. Reasonable system structure ensures the decision support system for mechanical conservation to be practiced. The system frame includes data layer, logic layer, component layer and function layer, and its details are in Fig. 3.

\subsection{System Development Platform}

This decision support system is developed on the platform of.NET Framework 2.0 and SQL Server 2005, which integrates three models, such as potential estimation model based on projection pursuit, adaptability estimation and model selection model based on fuzzy comprehensive evaluation, economic benefit estimation and sensitivity analysis model based on the time value of money. 
.NET Framework includes two main components: common language runtime library and class library. common language runtime library is a software engine used to load applications, confirm them to run without any errors, verify the security license, execute them and clear them when work is finished, and NET framework class library is a reusable type set that tight integrated with common language runtime library[7].

SQL Server defined an xml data type that could be used either as a data type in database columns or as literals in queries. SQL Server introduced a method of allowing usage of database connections for multiple purposes [8]. And it can be used to monitor the health of a server instance, diagnose problems, and tune performance. For relational data, SQL Server has been augmented with error handling features and support for recursive queries with common table expressions.

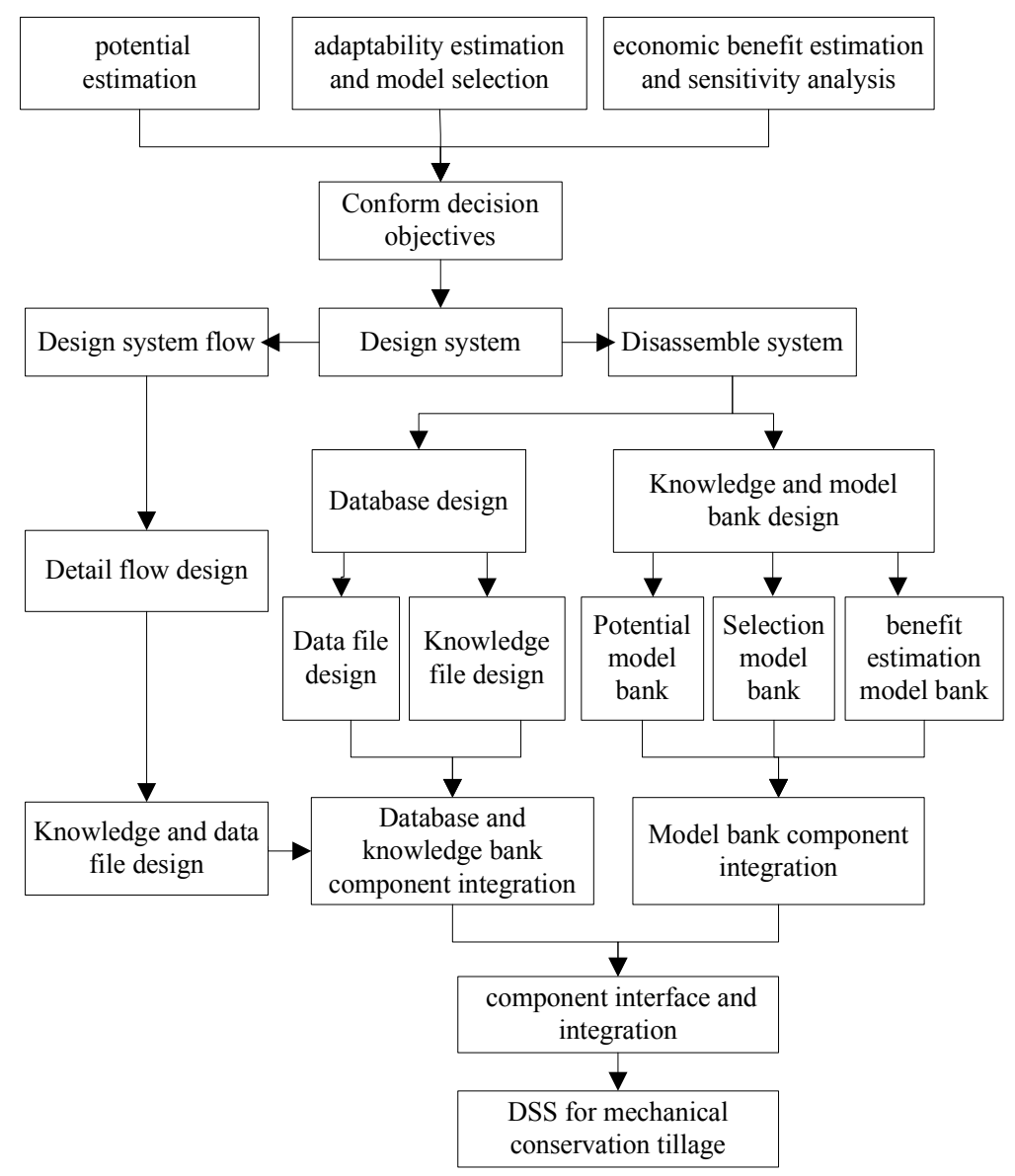

Fig. 2. Flow of decision support system development 

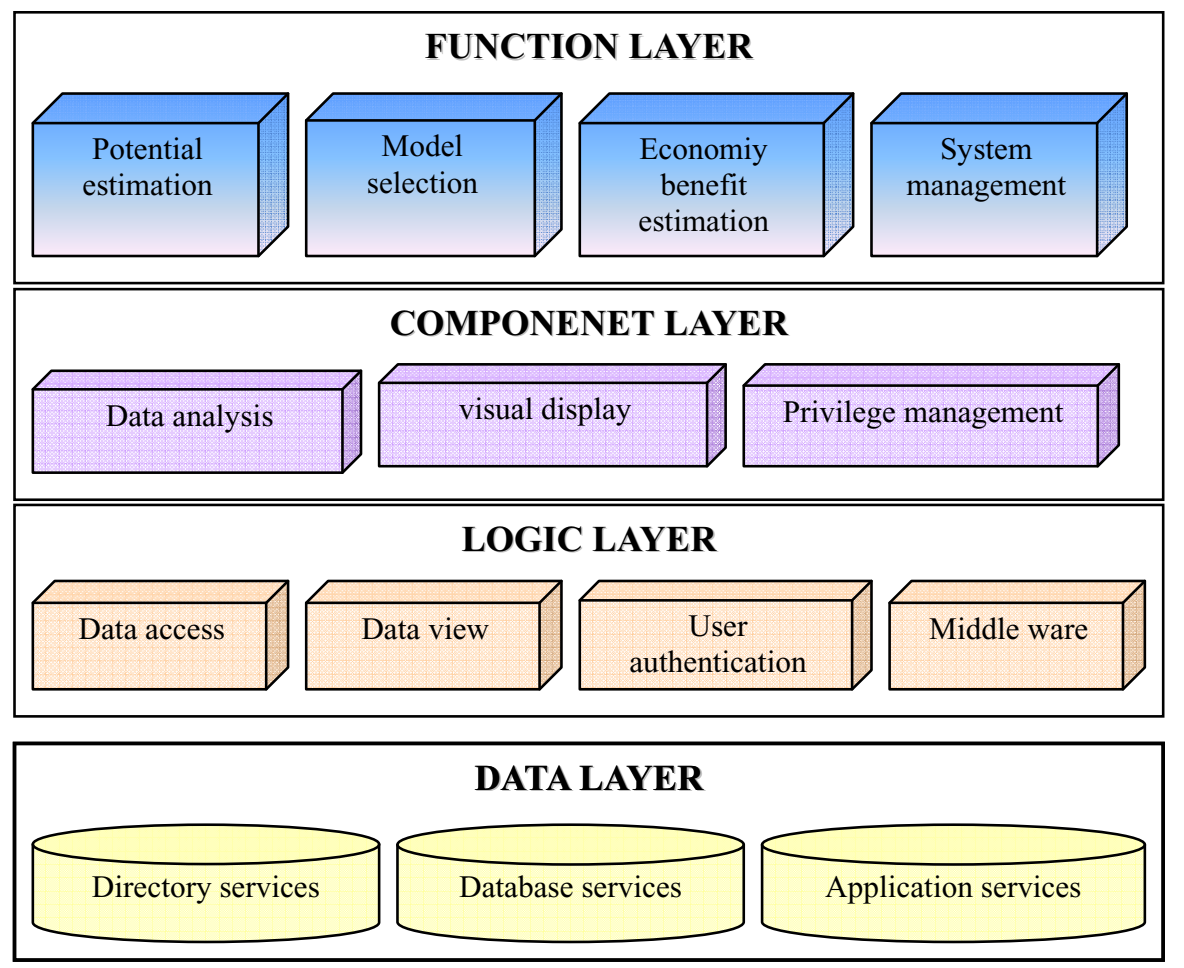

Fig. 3. Frame of decision support system

\section{Conclusion}

According to the above methods and steps, the author researches and develops the decision support system for mechanical conservation tillage, which is an available assistant tool to analyze data and information for the decision-maker. The system adopts .NET+ SQL Server which satisfies the demand functions, and the platform is in common use and easy to learn.

\section{References}

1. Zhang, G.: Different tillage influence on topsoil structure and organic carbon in cropland. Gan Su Agricultural University, College of Resources and Environmental Sciences, Gansu (2004) (in Chinese)

2. Lehmann, J., Kinyangi, J., Solomon, D.: Organic matter stabilization in soil microaggregates: implications from spatial heterogeneity of organic carbon contents and carbon forms. In: Biogeochemistry, Springer, Netherlands (2007)

3. Erm, C.: Decision Support System: A summary, problems and future trends. In: Decision support systems, vol. 4, pp. 355-363. Elsevier, Amsterdam (1988) 
4. Yi, P., Guo, Y., Hou, H.: A model-oriented decision-making support system. Journal of northeastern university 29, 292-296 (2008) (in Chinese)

5. Li, H., Gao, H., Zhou, X., Mao, N.: Economic analysis of maize production in dryland with conservation tillage (in Chinese). Agricultural Research in the Arid Areas, vol. 29. pp. 44-49. Yangling, Shanxi (2000)

6. Chen, Y., Li, D.: Web-Gis and RS based spatial decision support system for revegetation in coal mine waste area (in Chinese). Transactions of the CSAE 24, 63-67 (2008)

7. Brief introduction of .net framework, http://www.qqread.com/z/net_framework/2.html

8. Microsoft SQL Server,

http://en.wikipedia.org/wiki/Microsoft_SQL_Server 